\title{
Review: transcriptome and trans-omics analysis of systemic lupus erythematosus
}

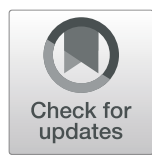

Keishi Fujio*, Yusuke Takeshima, Masahiro Nakano and Yukiko Iwasaki

\begin{abstract}
Systemic lupus erythematosus (SLE), which was recognized as a defined clinical entity more than 100 years ago, is an archetype for systemic autoimmune diseases. The 10-year survival of SLE patients has shown dramatic improvement during the last half-century. However, SLE patients receiving long-term prednisone therapy are at high risk of morbidity due to organ damage. Identification of key immune pathways is mandatory to develop a suitable therapy and to stratify patients based on their responses to therapy. Recently developed transcriptome and omic analyses have revealed a number of immune pathways associated with systemic autoimmunity. In addition to type I interferon, plasmablast and neutrophil signatures demonstrate associations with the SLE phenotype. Systematic investigations of these findings enable us to understand and stratify SLE according to the clinical and immunological features.
\end{abstract}

Keywords: Systemic lupus erythematosus, Transcriptome, Omics, eQTL

\section{Background}

Systemic lupus erythematosus (SLE) is an archetype of autoimmune disease. It is characterized by its predominance in woman of childbearing age $[1,2]$. Clinical heterogeneity, together with the potential severity of these manifestations, make the management of SLE a distinct challenge for rheumatologists. Despite recent progress in treatment protocols, several studies have pointed out that SLE patients still have an overall 2- to 3-fold increased risk of death [3, 4]. In this context, an initiative to evaluate possible therapeutic targets and develop treat-to-target guidance would be highly appropriate in the management of SLE patients.

The Asia Pacific Lupus Collaboration (APLC) proposed the existence of a Lupus Low Disease Activity State (LLDAS). This was subsequently defined operationally via a nominal consensus approach [5]. LLDAS was defined as follows: SLE Disease Activity Index (SLEDAI) $2 \mathrm{~K} \leqq 4$, with no activity in major organ systems; no new lupus disease activity compared with the

* Correspondence: kfujio-tky@umin.ac.jp

Department of Allergy and Rheumatology, Graduate School of Medicine, The University of Tokyo, 113-8655 7-3-1 Hongo, Bunkyo-ku, Tokyo, Japan previous assessment; SLEDAI Physician Global Assessment $1 \leqq 4$; a current prednisolone (or equivalent) dose $\leqq 7.5 \mathrm{mg}$ daily; and well-tolerated standard maintenance doses of immunosuppressive drugs. Patients who spent greater than $50 \%$ of their observed time in LLDAS had significantly reduced accrual of organ damage compared with patients who spent less than $50 \%$ of their time in LLDAS and were significantly less likely to have increased organ damage. However, some patients developed organ damage in spite of the maintenance of LLDAS. While some patients remain serologically active clinically quiescent (SACQ) indefinitely or become serologically quiescent clinically quiescent (SQCQ), others' SACQ periods are terminated by disease flares for which reliable predictors have yet to be identified [6]. Therefore, novel biomarkers are necessary to improve prognostic stratification, monitoring of treatment responses, and detection of early renal flares. Transcriptome and omics analyses, which evaluate the genome, transcriptome, and epigenome, are powerful approaches to the identification of clinically useful biomarkers.

(c) The Author(s). 2020 Open Access This article is licensed under a Creative Commons Attribution 4.0 International License, which permits use, sharing, adaptation, distribution and reproduction in any medium or format, as long as you give appropriate credit to the original author(s) and the source, provide a link to the Creative Commons licence, and indicate if changes were made. The images or other third party material in this article are included in the article's Creative Commons licence, unless indicated otherwise in a credit line to the material. If material is not included in the article's Creative Commons licence and your intended use is not permitted by statutory regulation or exceeds the permitted use, you will need to obtain permission directly from the copyright holder. To view a copy of this licence, visit http://creativecommons.org/licenses/by/4.0/. 


\section{Key molecular signatures in SLE}

SLE patients demonstrate transcriptional signatures related to type I interferon (IFN) and granulocytes [7, 8]. Although a number of studies have focused on the linkage between IFN-induced transcripts or proteins and SLE disease activity, most of them suffer from samplesize limitations and essential clinical and therapeutic heterogeneity in SLE. Chaussabel et al. applied modular analysis in PBMC microarray data from 239 individuals [9]. Differentially expressed gene (DEG) analysis tolerates noise due to the large number of comparisons performed (usually $>10,000$ ). In order to reduce noise, they focused the analysis on small sets of coordinately expressed transcripts that constitute coherent and biologically meaningful transcriptional units. Among 28 transcriptional modules linked with pathways or cell types involved in immune processes, two modules, IFNinducible and neutrophil genes, exhibited positive correlations with SLE disease activity, SLEDAI. In contrast, gene modules corresponding to ribosomal proteins and surface molecule of $\mathrm{T}$ cells including CD5, CD6, CD7, CD26, CD28, and CD96 negatively correlated with SLEDAI. Intriguingly, in one patient who showed disease flaring and subsequent recovery, an increase in the transcriptional score was detected 2 months before deterioration of the patient's clinical condition. Their following report also revealed complex IFN signatures in SLE that were composed of 3 modules and involved both an IFN$\alpha$ signature and those for IFN- $\beta$ and IFN- $\gamma$ [10].

The possibility of clinical application of IFN gene expression data has been demonstrated by a two-score system for IFN status. Factor analysis by El-Sherbiny et al. in 328 autoimmune disease patients reduced the 30 ISGs (interferon signature genes) to 2 factors, IFN-Score-A and IFN-Score-B [11]. Although IFN-Score-A and IFN-ScoreB shared several key ISGs, specific genes for IFN-Score-A were mostly typical ISGs, i.e., IFIT1, IFI27, RSAD2, IFI44L, IRF7, ISG15, and XAF1. On the other hand, specific genes for IFN-Score-B were LAMP3, UNC93B1, SP100, IFI16, IFIH1, PHF11, and BST2. IFN-Score-A differentiated SLE from both rheumatoid arthritis (RA) and healthy controls (HC), while IFN-Score-B differentiated SLE and RA from HC. In SLE, both scores were associated with cutaneous and hematological but not musculoskeletal disease activity. Yusof et al. examined these scores in 118 individuals at risk of autoimmune connective tissue diseases [12]. At baseline, both IFN scores were elevated in those at-risk who progressed to connective tissue diseases at 12 months versus nonprogressors, to a greater extent for IFN-ScoreB (3.22-fold difference) than IFN-Score-A (2.94-fold difference). The fold-difference between at-risk and HCs for IFN-Score-A was markedly greater in the skin than blood. These results suggested potential application of IFN-score in lupus patients.
Banchereau et al. reported a longitudinal modular study of 158 SLE patients in the Immunological Genome Project Consortium [13]. They found that positive correlates of disease activity were enriched for the IFN response as well as plasmablast, cell cycle, neutrophil, histone, and B cell modules. Notably, the plasmablast signature was the most reproducible, and plasmablast responses showed increases in African-American patients who presented with more severe disease than did Caucasians. In addition, the neutrophil, myeloid lineage, and inflammation modules were enriched in patients with lupus nephritis. These observations support a model of sequential disease progression, whereby an initial increase in IFN response and differentiation of B cells into short-lived plasmablasts, which may occur before clinical onset, is followed by lupus nephritis and full-blown systemic inflammation enhanced by myeloid cells, including neutrophils.

One limitation of transcriptome analysis of peripheral blood cells or PBMC is the distorting effects of variations in the size of each immune subset. That is, transcriptome analyses of a mixture of cell subsets are obscured by the variations in the frequencies of different cell types, as shown in comprehensive analyses in the human brain of bulk/single-cell transcriptomes, active enhancers, genotypes, and $\mathrm{Hi}-\mathrm{C}$ data sets, named PsychENCODE [14]. In PsychENCODE, weighted combinations of single-cell signatures could account for most of the population-level expression variation, with an accuracy of $>88 \%$. Therefore, more detailed analyses of immune cell subsets are required to advance modular findings obtained from peripheral blood or PBMC. McKinney et al. conducted a subset-specific transcriptomic analysis of 58 patients with ANCA-associated vasculitis (AAV) and 23 patients with SLE. They found that while $\mathrm{CD}^{+}{ }^{+} \mathrm{T}$ cell co-stimulation is pronounced, genes specifically downregulated in exhausted $\mathrm{CD}^{+} \mathrm{T}$ cells during chronic murine lymphocytic choriomeningitis virus (LCMV) infection were downregulated in $\mathrm{CD}^{+} \mathrm{T}$ cells from patients at low risk of relapse of AAV and SLE. This result suggested that whereas $\mathrm{CD}^{+}$exhaustion is associated with poor outcome in viral infection, it predicts favorable outcome in autoimmune diseases.

Shi et al. studied monocytes from 9 SLE patients using RNA-seq of both coding and noncoding RNAs [15]. They found that SLE patients had diminished expression of most endogenous retroviruses and small nucleolar RNAs. Conversely, lupus monocytes exhibited increased expression of pri-miRNAs and altered splicing patterns and polyadenylation. Rai et al. analyzed peripheral blood RNA-seq data from 28 SLE patients [16] to find that anti-dsDNA-positive patients showed dysregulation of multiple cytokine signaling pathways. In contrast, antiextractable nuclear antigen (ENA)-positive patients exhibited specific dysregulation of IFN signaling. 


\section{Approach to lupus nephritis}

Lupus nephritis is a major risk factor for overall morbidity and mortality in SLE. Kidney biopsy and histological evaluation is the gold standard to determine the optimal treatment protocol. Prediction of prognosis based upon histological classification is insufficient, and transcriptomic information may improve patients' stratification. Parikh et al. examined the expression of 511 immuneresponse genes with NanoString in kidney biopsies from 19 patients [17]. The top genes responsible for clinical response included several interferon pathway genes (STAT1, IRF1, IRF7, MX1, STAT2, JAK2), while complement genes $(C 1 R, C 1 Q B, C 6, C 9, C 5, M A S P 2)$ were mainly responsible for non-clinical response.

Single-cell RNA-seq is a powerful approach that enables investigators to comprehensively evaluate inflamed organs at single-cell resolution [18, 19]. Based on the transcriptional signatures of such immune cells, the data reveal the immunological nature of inflammation and novel biomarkers. The Accelerating Medicines Partnership (AMP) is a public-private partnership created to develop new ways of identifying and validating promising biological targets for diagnostics and drug development. Arazi et al. analyzed kidney samples from 24 patients with lupus nephritis and from 10 control subjects using single-cell RNA sequencing [20]. They identified 21 subsets of leukocytes in lupus nephritis, including multiple populations of myeloid cells, T cells, natural killer cells, and $\mathrm{B}$ cells that demonstrated both pro-inflammatory responses and inflammation-resolving responses. Similar to peripheral blood cells, almost all immune cell subsets in the kidney demonstrated elevation of type I interferon. In particular, local activation of B cells was consistent with an age-associated $B$ cell $(A B C)$ signature that contributes to cytokine production and antigen presentation [21]. Trajectory analysis identifies a continuum of intermediate states spanning patrolling, phagocytic, and alternatively activated monocytes, suggesting progressive stages of monocyte differentiation within the kidney.

Der et al. applied single-cell RNA-seq to kidney and skin biopsy samples from 16 lupus nephritis patients [22]. IFN signatures in tubular cells and keratinocytes were specific features of patients with LN. Moreover, a high IFN signature and extracellular matrix (ECM) fibrotic signature in tubular cells were each associated with resistance to therapy. When they proposed an equation predicting response to treatment at 6 months post-biopsy, four genes, COL1A1, COL14A1, COL1A2, and COL5A2, were found to significantly explain variance and predict response to treatment with a $92 \%$ accuracy. With greater sample sizes, their analysis may help to identify disease-relevant or disease subtyperelated populations that only exist in affected organs.

\section{Combining transcriptomics and genetic analysis}

Genome-wide association study (GWAS) revealed a number of single nucleotide polymorphisms (SNPs) and genes associated with SLE pathogenesis [23]. GWAS has successfully identified more than 90 SLE risk loci that explain a significant proportion of SLE's heritability. GWAS-SNP genes suggest several pathogenic pathways in SLE such as cytokine pathways including immune complex processing and phagocytosis, DNA degradation, neutrophil, and monocytes signaling; Toll-like receptor and/or type I interferon signaling; nuclear factor kappa beta (NF-KB) activation; and $\mathrm{B}$ and $\mathrm{T}$ cell function and signaling [24-26]. However, one of the great challenges posed by interpreting GWAS data is determining the causal genes implicated by the genetic association data. The association of a locus with disease does not specify which gene is affected by the causal variant. The fact that $>90 \%$ of disease-associated variants are located in non-protein-coding regions of the genome suggests that many loci implicated by GWAS alter the genetic regulation of one or more target genes. Therefore, combinatory analysis using both transcriptomics and genetic analysis may precisely reveal target genes for diseaserelated SNP and detailed immunological pathogenesis.

Zhang et al. simultaneously obtained RNA-seq and H3K4me3 Chip-seq data in T cells, B cells, and monocytes from 6 SLE patients [27]. Across the 3 cell types, there was $55 \%$ concordance for gene expression changes related to SLE. Key conserved pathways were ribosome biogenesis among upregulated genes and heat shock response among downregulated genes. Upon exploration of a common pathway linking H3K4me3 and gene expression, ATF3 showed a significant association across all cell types in both RNA-seq and ChIP-seq data sets. ATF3 is inducible by type I interferons in macrophages, providing a potential mechanism for increased function in SLE. When they compared the change in expression and change in H3K4me3 near the GWAS variants, there was a decrease in RNA abundance in all three cell types near the GWAS variants. With regard to H3K4me3, monocytes exhibited decreased H3K4me3 in GWAS variant region. These data are consistent in supporting the concept that GWAS variants impact gene regulation.

Genetic and epigenetic fine mapping has shown that most of the immune-mediated disease (IMD)-related SNPs are located within enhancer regions [28]. As enhancers regulate expression of target genes, SNPs in enhancer regions affect the expression levels of target genes. This effect, called expression quantitative trait locus (eQTL), provides important information regarding the function and role of disease-related SNPs and genes [29]. Bentham et al. performed large-scale GWAS comprised 7219 cases and 15,991 controls of European ancestry, combined with eQTL analysis using monocytes 
and B cells transcriptome data from database [23]. They identified eQTL effects and candidate causal genes in each immune cell subset and found an overrepresentation of 16 transcription factors among SLE susceptibility genes. However, there was a limitation that transcriptome data was not derived from SLE patients. Panousis et al. combined genotype and RNA-sequencing data to comprehensively evaluate blood transcriptomes in 142 SLE patients [30]. As anticipated, principal component analysis (PCA) discriminated transcriptomes in clinically active, inactive patients, and healthy individuals. On the other hand, inactive SLE cases were differentiated from healthy but not from active SLE, denoting persistently deregulated gene expression despite remission. Therefore, they determined a "susceptibility" signature that persists in the absence of disease activity following treatment. These genes were enriched in regulation and response of the immune system processes suggesting the persistence of immune activation. Notably, a total of 365 DEGs from the inactive versus active SLE comparison that are not in the "susceptibility" signature were enriched for oxidative phosphorylation, ribosomes, and cell cycle. With regard to organ damage, neutrophil activation and humoral response signatures correlated with lupus nephritis. Furthermore, Panousis et al. evaluated by eQTL analysis the extent to which transcriptomic changes in SLE may be genetically determined. eQTL analysis revealed 9 genes where both GWAS and eQTL identified the same variant, namely $U B E 2 L 3$, HLA-DRB5, RP11-356I2.3, BLK, FAM167A, NADSYN1, RP11-660 L16.2, $A L D H 2$, and $A L D H 18 A 1$, thus implicating them in SLE pathogenesis.

Cytokines play central roles in the network of systemic autoimmunity. Davenport et al. examined interactions between eQTLs and cytokine levels in a lupus clinical trial to define target genes and mechanisms [31]. They conducted whole-blood RNA-seq in subjects who had or had not been treated with anti-IL6 at 0,12 , and 24 weeks of treatment. eQTL analysis of 379 samples from 157 patients revealed 4818 cis eQTL genes. When they classified each sample as either IFN high or IFN low using real-time PCR of 11 IFNinducible genes, they identified 210 eQTLs which effect is magnified in IFN-high samples. Similarly, they identified 121 eQTLs which effect is magnified under anit-IL-6 drug treatment. Biologically relevant anti-IL-6 drug-eQTL interactions were noted for IL10, an anti-inflammatory cytokine, $C L E C 4 C$, which has previously been associated in trans with an SLE risk allele, and CLEC18A, another member of the C-type lectin domain family. Notably, the IRF4 motif was enriched among eQTLs which showed enhanced effect under anti-IL-6 drug treatment, suggesting that the interruption of transcription factor binding motifs by eQTL interaction SNPs may be a key regulatory mediator of environmental stimuli and potential therapeutic targets.

\section{T cell receptor and B cell receptor repertoires}

Autoreactive $\mathrm{T}$ cells play critical roles in organ inflammation and promotion of autoantibody development. $\mathrm{T}$ cell receptors (TCR) that recognize autoantigens are generated by genomic rearrangement of the variable $(\mathrm{V})$, diversity (D), and joining (J) regions. Expansion of the partial TCRs and reduction of TCR repertoire diversity were observed in peripheral blood cells of patients diagnosed with autoimmune disease [32]. RNA-seq effectively reveals the detailed nature of the TCR repertoire. Liu et al. examined the TCR repertoire using RNA-seq in peripheral blood cells obtained from 877 SLE patients, 206 RA patients, and $439 \mathrm{HCs}$ [33]. Significant differences were identified in $\mathrm{V}, \mathrm{J}$, and $\mathrm{V}-\mathrm{J}$ pairing among the SLE or RA and HC groups. Intriguingly, the 3 groups were almost perfectly discriminated by these differences. Consistent with previous studies reporting the importance and antigenic association of public TCRs in various diseases, TCR clones with a higher publicity in SLE and RA had higher clonal frequencies compared with HCs. They identified 198 SLEassociated TCR clones and 53 RA-associated clones. Moreover, in SLE, the Gini index of the V-J gene combinations and the content of SLE-associated clones were positively correlated with SLEDAI. The content of SLEassociated TCR clones and the total unique clone number were correlated with complement C3 and C4.

As is the case with the TCR, understanding of the BCR repertoire in the context of IMD could provide us new insights into pathogenesis and therapy. Bashford-Rogers et al. compared the BCR repertoire in SLE, AAV, Crohn's disease, Behçet's disease, eosinophilic granulomatosis with polyangiitis, and immunoglobulin A (IgA) vasculitis [34]. The BCR repertoire of SLE showed an increase in clonality and a dominance of the IgA isotype, suggesting a microbial contribution to disease. When the effect of immunosuppressive therapy was evaluated, MMF (mycophenolate mofetil) increased the proportion of $\operatorname{IgM}^{+}$and $\operatorname{IgD}^{+} \mathrm{B}$ cells and reduced the number of isotype-switched $\mathrm{B}$ cells. Conversely, after rituximab treatment in SLE, the number of circulating B cells was low but persisting cells were largely isotype-switched and clonally expanded IgG1- or IgG2expressing cells. These characteristics of the TCR and BCR repertoire, particularly the disease-associated clones, may become useful biomarkers.

\section{Conclusion}

Transcriptomic and omics analyses have revealed several important features of immunity in SLE. (1) IFNinducible, neutrophil, and plasmablast genes positively correlate with SLE disease activity and lupus nephritis. (2) IFN scores predict the development of systemic autoimmune diseases. (3) CD8 exhaustion predicts favorable outcome in autoimmune diseases. (4) B cells with an $\mathrm{ABC}$ phenotype infiltrate and monocytes progressively 
differentiate in lupus kidneys. ECM fibrotic signature in tubular cells is associated with resistance to therapy. (5) Public TCR in SLE is highly disease-specific, and the BCR repertoire of SLE showed an increase in clonality and dominance of an IgA isotype. This finding will help us to understand the pathogenesis of lupus and to stratify essentially heterogenous SLE patients.

\section{Abbreviation}

SLE: Systemic lupus erythematosus; LLDAS: Lupus Low Disease Activity State; IFN: Interferon; DEG: Differentially expressed gene; SACQ: Serologically active clinically quiescent; SLEDAI: SLE Disease Activity Index; AAV: ANCA-associated vasculitis; GWAS: Genome-wide association study; SNPs: Single nucleotide polymorphisms; IMD: Immune-mediated disease; eQTL: Expression quantitative trait locus; PCA: Principal component analysis; TCR: T cell receptors

\section{Acknowledgements}

None

\section{Authors' contributions}

K.F, Y.T, N.M, and Y.I. contributed to the writing of this manuscript. The authors read and approved the final manuscript.

\section{Funding}

Not applicable

\section{Availability of data and materials \\ Not applicable}

\section{Ethics approval and consent to participate}

Not applicable

\section{Consent for publication}

Not applicable

\section{Competing interests}

The author(s) declare(s) that they have no competing interests.

Received: 25 March 2020 Accepted: 8 June 2020

Published online: 18 June 2020

\section{References}

1. Nagafuchi Y, Shoda H, Fujio K: Immune profiling and precision medicine in systemic lupus erythematosus. Cells 2019, 8(2).

2. Takeshima Y, Iwasaki Y, Fujio K, Yamamoto K. Metabolism as a key regulator in the pathogenesis of systemic lupus erythematosus. Semin Arthritis Rheum. 2019:48(6):1142-5.

3. Thomas G, Mancini J, Jourde-Chiche N, Sarlon G, Amoura Z, Harle JR, et al. Mortality associated with systemic lupus erythematosus in France assessed by multiple-cause-of-death analysis. Arthritis Rheumatol. 2014;66(9):2503-11.

4. Yee CS, Su L, Toescu V, Hickman R, Situnayake D, Bowman S, et al. Birmingham SLE cohort: outcomes of a large inception cohort followed for up to 21 years. Rheumatology (Oxford). 2015;54(5):836-43.

5. Franklyn K, Lau CS, Navarra SV, Louthrenoo W, Lateef A, Hamijoyo L, et al, Definition and initial validation of a lupus low disease activity state (LLDAS). Ann Rheum Dis. 2016;75(9):1615-21.

6. Steiman AJ, Gladman DD, Ibanez D, Urowitz MB. Prolonged serologically active clinically quiescent systemic lupus erythematosus: frequency and outcome. J Rheumatol. 2010:37(9):1822-7.

7. Bennett L, Palucka AK, Arce E, Cantrell V, Borvak J, Banchereau J, et al. Interferon and granulopoiesis signatures in systemic lupus erythematosus blood. J Exp Med. 2003;197(6):711-23.

8. Baechler EC, Batliwalla FM, Karypis G, Gaffney PM, Ortmann WA, Espe K et al. Interferon-inducible gene expression signature in peripheral blood cells of patients with severe lupus. Proc Natl Acad Sci U S A. 2003;100(5): 2610-5.
9. Chaussabel D, Quinn C, Shen J, Patel P, Glaser C, Baldwin N, et al. A modular analysis framework for blood genomics studies: application to systemic lupus erythematosus. Immunity. 2008;29(1):150-64.

10. Chiche L, Jourde-Chiche N, Whalen E, Presnell S, Gersuk V, Dang K, et al. Modular transcriptional repertoire analyses of adults with systemic lupus erythematosus reveal distinct type I and type II interferon signatures. Arthritis Rheumatol. 2014;66(6):1583-95.

11. El-Sherbiny YM, Psarras A, Md Yusof MY, Hensor EMA, Tooze R, Doody G, et al. A novel two-score system for interferon status segregates autoimmune diseases and correlates with clinical features. Sci Rep. 2018. 8(1):5793.

12. Md Yusof MY, Psarras A, El-Sherbiny YM, Hensor EMA, Dutton K, UI-Hassan S, et al. Prediction of autoimmune connective tissue disease in an at-risk cohort: prognostic value of a novel two-score system for interferon status. Ann Rheum Dis. 2018:77(10):1432-9.

13. Banchereau R, Hong S, Cantarel B, Baldwin N, Baisch J, Edens M, et al. Personalized immunomonitoring uncovers molecular networks that stratify lupus patients. Cell. 2016;165(6):1548-50.

14. Wang D, Liu S, Warrell J, Won H, Shi X, Navarro FCP, et al. Comprehensive functional genomic resource and integrative model for the human brain. Science. 2018:362(6420).

15. Shi L, Zhang Z, Yu AM, Wang W, Wei Z, Akhter E, et al. The SLE transcriptome exhibits evidence of chronic endotoxin exposure and has widespread dysregulation of non-coding and coding RNAs. PLoS One. 2014 9(5):e93846.

16. Rai R, Chauhan SK, Singh W, Rai M, Rai G. RNA-seq analysis reveals unique transcriptome signatures in systemic lupus erythematosus patients with distinct autoantibody specificities. PLoS One. 2016;11(11):e0166312.

17. Parikh SV, Malvar A, Song H, Alberton V, Lococo B, Vance J, et al. Characterising the immune profile of the kidney biopsy at lupus nephritis flare differentiates early treatment responders from non-responders. Lupus Sci Med. 2015;2(1):e000112.

18. Stubbington MJT, Rozenblatt-Rosen O, Regev A, Teichmann SA. Single-cell transcriptomics to explore the immune system in health and disease. Science. 2017;358(6359):58-63.

19. Papalexi E, Satija R. Single-cell RNA sequencing to explore immune cell heterogeneity. Nat Rev Immunol. 2018;18(1):35-45.

20. Arazi A, Rao DA, Berthier CC, Davidson A, Liu Y, Hoover PJ, et al. The immune cell landscape in kidneys of patients with lupus nephritis. Nat Immunol. 2019;20(7):902-14.

21. Jenks SA, Cashman KS, Zumaquero E, Marigorta UM, Patel AV, Wang X, et al. Distinct effector B cells induced by unregulated toll-like receptor 7 contribute to pathogenic responses in systemic lupus erythematosus. Immunity. 2018;49(4):725-39 e726.

22. Der E, Suryawanshi H, Morozov P, Kustagi M, Goilav B, Ranabothu S, et al. Tubular cell and keratinocyte single-cell transcriptomics applied to lupus nephritis reveal type I IFN and fibrosis relevant pathways. Nat Immunol. 2019;20(7):915-27

23. Bentham J, Morris DL, Graham DSC, Pinder CL, Tombleson P, Behrens TW, et al. Genetic association analyses implicate aberrant regulation of innate and adaptive immunity genes in the pathogenesis of systemic lupus erythematosus. Nat Genet. 2015;47(12):1457-64.

24. Mohan C, Putterman C. Genetics and pathogenesis of systemic lupus erythematosus and lupus nephritis. Nat Rev Nephrol. 2015;11(6):329-41.

25. Tsokos GC, Lo MS, Costa Reis P, Sullivan KE. New insights into the immunopathogenesis of systemic lupus erythematosus. Nat Rev Rheumatol. 2016;12(12):716-30

26. Chen L, Morris DL, Vyse TJ. Genetic advances in systemic lupus erythematosus: an update. Curr Opin Rheumatol. 2017;29(5):423-33.

27. Zhang Z, Shi L, Song L, Maurer K, Petri MA, Sullivan KE. Overall downregulation of mRNAs and enrichment of $\mathrm{H} 3 \mathrm{~K} 4 \mathrm{me} 3$ change near genome-wide association study signals in systemic lupus erythematosus: cell-specific effects. Front Immunol. 2018;9:497.

28. Farh KK, Marson A, Zhu J, Kleinewietfeld M, Housley WJ, Beik S, et al. Genetic and epigenetic fine mapping of causal autoimmune disease variants. Nature. 2015:518(7539):337-43.

29. Ishigaki K, Kochi Y, Suzuki A, Tsuchida Y, Tsuchiya H, Sumitomo S, et al. Polygenic burdens on cell-specific pathways underlie the risk of rheumatoid arthritis. Nat Genet. 2017;49(7):1120-5.

30. Panousis NI, Bertsias GK, Ongen H, Gergianaki I, Tektonidou MG, Trachana M, et al. Combined genetic and transcriptome analysis of patients with SLE: 
distinct, targetable signatures for susceptibility and severity. Ann Rheum Dis. 2019;78(8):1079-89.

31. Davenport EE, Amariuta T, Gutierrez-Arcelus M, Slowikowski K, Westra HJ, Luo $Y$, et al. Discovering in vivo cytokine-eQTL interactions from a lupus clinical trial. Genome Biol. 2018;19(1):168.

32. Sakurai K, Ishigaki K, Shoda H, Nagafuchi Y, Tsuchida Y, Sumitomo S, et al. HLA-DRB1 shared epitope alleles and disease activity are correlated with reduced $T$ cell receptor repertoire diversity in CD4+ T cells in rheumatoid arthritis. J Rheumatol. 2018;45(7):905-14.

33. Liu X, Zhang W, Zhao M, Fu L, Liu L, Wu J, et al. T cell receptor beta repertoires as novel diagnostic markers for systemic lupus erythematosus and rheumatoid arthritis. Ann Rheum Dis. 2019;78(8):1070-8.

34. Bashford-Rogers RJM, Bergamaschi L, McKinney EF, Pombal DC, Mescia F, Lee JC, et al. Analysis of the B cell receptor repertoire in six immunemediated diseases. Nature. 2019:574(7776):122-6.

\section{Publisher's Note}

Springer Nature remains neutral with regard to jurisdictional claims in published maps and institutional affiliations.

Ready to submit your research? Choose BMC and benefit from:

- fast, convenient online submission

- thorough peer review by experienced researchers in your field

- rapid publication on acceptance

- support for research data, including large and complex data types

- gold Open Access which fosters wider collaboration and increased citations

- maximum visibility for your research: over $100 \mathrm{M}$ website views per year

At BMC, research is always in progress.

Learn more biomedcentral.com/submissions 